\title{
L'utopia della comunicazione: cibernetica e arte informale
}

\author{
Eduardo Grillo \\ Dottore di ricerca in Semiotica e comunicazione simbolica - Università di Siena \\ Cultore della materia in Semiotica - Università di Perugia \\ eduardogrillo79@gmail.com
}

\begin{abstract}
Philippe Breton's appealing theory (L'utopie de la communication) places the birth of the communication society in the Forties of the last century, and recognises it as a post-traumatic value, come to light following the horrors of World War II. The cybernetic scientists, Breton's book main characters, actually insisted on proposing a notion of information which is able to reduce the communication ambiguities. The model is the machine, indifferent towards the irrational tendencies of humans. The man itself needs to become transparent and fully communicating in order to guarantee the social self-regulation. Informal artists are motivated by the same concerns and by the same interest in communication, but through the expression of an alternative model: the conversation - actually an indefinite and informal form. The uncertain and vague artistic forms of the period can be seen as an invitation to an open dialogue. As long as the typical utopian season of the immediate post-war period lasted, communication has been inserted within the production process and put at the consumer culture disposal. Then the informal disappeared, replaced by Pop Art, which has put under the spotlight the new protagonists of history: the serial object and our relationship with it.
\end{abstract}

\section{Parole chiave}

Secondo dopoguerra, Informale, utopia, comunicazione, modello conversazionale

\section{Key Words}

Post WWII, Informal Art, Utopia, Communication, Conversational Model

\section{Sommario}

1. Introduzione

2. Il Dopoguerra, l'impresa cibernetica e l'utopia della comunicazione

3. La mossa dell'Informale

4. La fine dell'utopia e la Pop Art

Bibliografia 


\section{Introduzione}

Philippe Breton (1992) ha suggerito con forza che il Secondo Dopoguerra abbia rappresentato un punto di svolta tra il prolungamento della società ottocentesca nel XX Secolo e l'inizio dell'era della comunicazione. Infatti, per il sociologo francese l'atto di nascita dell'attuale società della comunicazione va rintracciato nella tragedia dello sterminio degli ebrei, che viene confermato ufficialmente nel 1942. Allo stesso tempo, alcuni scienziati in America danno vita al progetto cibernetico, sotto la guida del matematico Norbert Wiener. Se i due fatti sono noti, non è chiaro il modo in cui essi possano essere messi in relazione.

L'idea di Breton è la seguente: gli uomini e gli scienziati dell'epoca attribuirono la responsabilità di tali tragici eventi alla segretezza che li avvolse. Inoltre, essi assunsero che il carattere radicalmente irrazionale di una tale diffusa strategia, sebbene perfettamente organizzata, fosse dovuta all'intrinseca irrazionalità dei fini e delle azioni umane. Questo spiegherebbe l'insistenza sia sui processi comunicativi, sia sulle macchine: l'ideale di uomo futuro che guidava l'impresa degli scienziati consisteva in un essere sempre comunicante, perfettamente trasparente, disposto a cedere sempre più spazio nei processi decisionali alle razionalissime macchine.

L'ipotesi è indubbiamente interessante: la comunicazione assumerebbe il carattere di "valore post-traumatico", in grado di orientare gli sforzi degli scienziati verso la formazione di una vera e propria utopia. Si può discutere sulla bontà dell'interpretazione; lo stesso Paolo Fabbri, nell'introdurre la traduzione italiana (1995) prende le distanze, suggerendo una più attenta ispezione della moltiplicazione dei media nel tardo Ottocento o sulla macchina propagandistica del New Deal (tra parentesi, ancora un contesto post-traumatico). Non voglio negare la natura controversa dell'ipotesi; oltretutto, altri studiosi (tra gli altri Kline 2015; Heims 1991) hanno fornito dei quadri interpretativi dell'avventura cibernetica più approfonditi e variegati. Tuttavia, mi interessa svolgere l'ipotesi di Breton fino in fondo per due ragioni: 1) sembra individuare l'aspetto della cibernetica che ha avuto maggior impatto nel corpo sociale; anche per questo, 2) risulta molto efficace nel fare emergere similarità e differenze tra alcuni orientamenti scientifici e molte esperienze artistiche coeve. Pertanto, il presente contributo non si pone l'obiettivo di un'analisi complessiva del fenomeno cibernetico, né di indagare a fondo la plausibilità storico-sociale dell'ipotesi di Breton. Si tratta invece di utilizzare una lente di ingrandimento in grado di mettere in risalto alcune linee di tendenza.

$\mathrm{E}$ in effetti, assumendo la prospettiva di Breton si evidenzia un curioso divaricamento tra le soluzioni tecno-sociali tipiche del movimento cibernetico e quelle, formali, tentate dagli artisti. Potrebbe sembrare che l'arte informale, esplosa proprio a partire dagli anni '40, abbia seguito linee di sviluppo decisamente "anti-comunicative", rielaborando alcuni portati della fisica coeva, 
Eduardo Grillo | L'utopia della comunicazione

che non tendevano affatto verso l'ordine espressivo e la trasparenza, ma al contrario percorrevano la strada dell'indeterminazione. In realtà, è possibile immaginare che l'Informale abbia condiviso con la nascente utopia della comunicazione le preoccupazioni, generate anch'esse dall'impatto degli orrori del recente conflitto, ma elaborando un modello comunicativo non informazionale, come quello di marca cibernetica, ma piuttosto conversazionale, puntando tutto sul dialogo in tempo reale, aperto e paritario, con i fruitori.

L'ipotesi sembra convincere anche in relazione al progressivo esaurirsi della stagione informale. A partire dagli anni '6o, la cultura sembra arrendersi alle impetuose dinamiche industriali; contemporaneamente l'emergente Pop Art sfrutta gli oggetti che circolano nell'universo dei media e della produzione di massa, con una sostanziale ri-presentazione e reduplicazione della loro invadenza. Da quel momento la centralità della comunicazione cambia radicalmente di segno, sancendo la fine della tensione utopistica.

\section{Il Dopoguerra, l'impresa cibernetica e l'utopia della comunica- zione}

Breton si mostra impudente e deciso nell'individuare il momento dell'avvento della società della comunicazione: il 1942. Esso è infatti un anno di svolta non soltanto per gli esiti della guerra che infuria per il mondo, ma anche da un punto di vista morale: è «il momento preciso in cui la barbarie organizzata riemerge proprio nel cuore della modernità» (Breton 1992: 61 tr. it.).

Infatti, per parte loro gli Alleati decidono di ricorrere senza più ambagi al bombardamento sulle popolazioni civili, mentre il carattere ideologico dell'offensiva nazista si fa quasi esclusiva, accentuando la propaganda anti-socialista e intensificando le strategie collegate alla "soluzione finale". La politica razziale nazista conosce un progressivo inasprimento; l'ultimo stadio, l'omicidio collettivo dei deportati, è accompagnato da un intensificarsi delle misure di segretezza. Sappiamo bene quanto le manovre naziste fossero conosciute dal popolo tedesco e dai governi stranieri; eppure, la piena consapevolezza dell'orrore che si stava consumando sul territorio del Terzo Reich giunse quasi a ciel sereno, proprio nel 1942 .

Agli occhi dei contemporanei, la segretezza e la razionalità ideologizzata furono i caratteri dominanti dell'orrore nazista; da qui gli sforzi della comunità scientifica, e in particolare dal gruppo cibernetico, di trovare un antidoto agli effetti devastanti di questi due "mostri" sociali. Entra in scena dunque la comunicazione, secondo un modello desunto dall'interazione delle macchine.

Difatti, la cibernetica fu un'iniziativa interdisciplinare che aveva lo scopo di unificare sotto un unico paradigma una serie di fenomeni già noti, caratterizzati da una qualità comunicativa, che riguardavano la neurofisiologia, l'elettronica, l'antropologia, in vista sia di applicazioni concrete, sia di una loro comprensione globale. L'ambizione era di costruire dei modelli generali in grado di trovare le "leggi" che governano ogni processo comunicativo, nelle interazioni tra macchine, tra gli animali e tra gli uomini. Fornire una definizione univoca di cibernetica è impossibile, considerate non soltanto la diversità degli intenti, ma anche la varietà delle posizioni in campo. Tuttavia, kybernētiké 
Eduardo Grillo | L'utopia della comunicazione

téchnē era l'arte di pilotare, di condurre un'imbarcazione, da kybernáō, ossia "io governo una nave".

Questo calco dal greco fu scelto proprio da Norbert Wiener, il padre della cibernetica, per dar un nome alla "scienza del controllo e della comunicazione". "Controllo" è la parola chiave: «Per Wiener, dunque, ogni riflessione sulla comunicazione è associata all'idea di regolazione, di comando e di padronanza» (Breton 1992: 17 tr. it.). L'inventore di questo nuovo campo di studi nutriva infatti la convinzione, condivisa dai suoi sodali, che intervenendo sui meccanismi comunicativi fosse possibile orientare i comportamenti collegati. Come diretta conseguenza, la cibernetica segue almeno due linee di sviluppo principali: la prima è quella delle ricadute scientifico-tecnologiche delle ricerche; la seconda, direttamente connessa con il problema della regolazione, è la riflessione generale sulle interazioni sociali che emerge una volta assunta la comunicazione come carattere chiave dei fenomeni umani.

Può colpire che un matematico di formazione e professione potesse avere tali ambizioni; ma Wiener concepiva la matematica in modo peculiare. Infatti, per Wiener la matematica è una "scienza di relazioni" (Cfr. Wiener 1948); e il mondo, naturale e sociale, è costituito dalle relazioni, dagli scambi e dalla circolazione delle informazioni: «Per Wiener, dunque, il mondo consiste interamente di "mediazioni": il punto di partenza del suo pensiero, anche di quello scientifico, è probabilmente questa "illuminazione mediologica"» (Breton 1992: 20 tr. it.). La matematica, e le sue applicazioni, erano pertanto legittimate a studiare ogni aspetto della vita sociale. Un tale approccio ha la conseguenza di elaborare una nuova definizione di "uomo".

Affermare che tutto può essere spiegato in termini di relazioni implica chiaramente che tutto sia "all'esterno" e che non ci sia il "resto". Ogni fenomeno oppure ogni "essere" è come una cipolla [...] La cipolla non possiede interiorità, è interamente costituita da esteriorità sovrapposte; togliete l'involucro esterno, ne incontrerete un altro e così via. [...] la "trasparenza" viene promossa al centro della ribalta. (Breton 1992, p. 23 tr. it.)

Si tratta dell'immagine di un uomo comunicante, implicato in una serie di scambi materiali e immateriali con l'ambiente che lo circonda, i quali definiscono in modo esaustivo la sua natura.

Il ruolo che la comunicazione è chiamata a giocare nello "schema cibernetico" allargato di Wiener è dunque «di combattere al tempo stesso il disordine generato dall'uomo e il male che la natura porta con sé» (Breton 1992: 29 tr. it.). Il nemico è pertanto l'entropia, quella tendenza verso il disordine e la disorganizzazione che trascina inesorabilmente i destini della natura.

In questo contesto è possibile solo un'azione locale, perché l'entropia resta una forza invincibile a livello globale. Ma ciò, invece di sollevare l'uomo dalle sue responsabilità, lo investe di un imperativo morale, poiché se già la natura tende al "male", ogni azione umana che non operi per il "bene" è correa della futura distruzione. Ora, il contrario dell'entropia, questo mostro informe ove tutto è equiprobabile, è appunto l'informazione, che struttura ordinatamente il mondo in cui opera. 
Eduardo Grillo | L'utopia della comunicazione

In questa prospettiva, l'informazione non è tanto ciò che viene detto, ma ciò che può esser detto. In altri termini, l'informazione è la misura di una possibilità di scelta nella selezione di un messaggio. Un messaggio computabile in 1 bit (la scelta tra due possibilità equiprobabili) e uno computabile in 3 bit (la scelta tra 8 possibilità equiprobabili), si distinguono per il numero di possibili scelte che la seconda situazione presentava - alla fonte - rispetto alla prima. Nel secondo caso il messaggio informa di più perché - alla fonte - c'era maggiore incertezza circa la scelta che sarebbe stata attuata. Attraverso l'adozione di un codice, che disciplina le scelte possibili, si perde informatività, ma si guadagna in precisione e trasmissibilità (Cfr. Eco 1972, Introduzione).

La "cura" dell'informazione ha quindi l'effetto di dare a coloro che se ne servono la possibilità dell'autoregolazione. Se i canali di comunicazione saranno sempre aperti e "puliti", nessuna distorsione disturberà la trasmissione e l'elaborazione dell'informazione e, secondo l'ottimista Wiener, la società umana avrà i mezzi per orientare la propria condotta.

Da questo punto di vista, l'uomo perde buona parte dei privilegi che si era assegnato; un partner comunicativo sufficientemente complesso si candida ad esser considerato un essere sociale. È la vita stessa, nella visione cibernetica, a passare dal dominio biologico a quello "comunicativo". Per rendersi conto della radicalità del pensiero antropologico di Wiener, è opportuno cedergli la parola per esteso:

L’identità fisica di un individuo non consiste della materia di cui egli è formato. [...] l'individualità del corpo è piuttosto quella di una fiamma che quella di una pietra, quella di una forma anziché quella di un frammento di sostanza. Questa forma può essere trasmessa e riprodotta, sebbene oggi noi conosciamo il modo per riprodurla su distanze brevi. [...] non vi è alcuna diversità fondamentale di carattere assoluto fra $\mathrm{i}$ tipi di trasmissione che possiamo impiegare per l'invio di un telegramma da un paese all'altro e i tipi di trasmissione che, almeno teoricamente, sono applicabili a un organismo vivente come l'uomo. Riconosciamo dunque che la vecchia idea del bambino, secondo il quale oltre a viaggiare in aereo o in treno si potrebbe presumibilmente viaggiare per telegrafo, non intrinsecamente assurda per quanta possa essere lontana dalla realizzazione. (Wiener 1950: 40 tr. it.) ${ }^{1}$

Non c'è alcun residuo oltre la comunicazione; l'uomo, e i suoi possibili partner (il candidato più probabile è il computer), è tutto lì. Si tratta di un anello intermedio, senza privilegi, della grande catena informazionale che costituisce la "società". In quanto fatto esclusivamente di informazione, l'uomo è dunque totalmente trasferibile, il che implica che sia manipolabile e utilizzabile; e

1 Sono evidenti le analogie con le riflessioni di Bateson, in particolare (Bateson 1979). Tuttavia, la compatibilità degli interessi di Bateson e del resto del movimento cibernetico è solo parziale. Mentre con Wiener c'erano almeno alcuni intenti comuni, con Von Neumann, ad esempio, i rapporti erano piuttosto conflittuali; si veda a questo proposito (Heims 1977). Oltre a ciò, alcuni aspetti del lavoro di Bateson si distaccano dalla maggior parte dei cibernetici, anticipando in qualche modo la cosiddetta "seconda cibernetica", meno fondata sulle nozioni di controllo e feedback negativo e più propensa a considerare l'autoriflessività dei sistemi viventi. 
Eduardo Grillo | L'utopia della comunicazione

«ogni "uso" dell'uomo che non utilizzi appieno questa facoltà fondamentale è degradante» (Breton 1992: 7 tr. it.).

Ma se l'uomo consiste interamente nel suo rapporto con gli altri esseri sociali e con l'ambiente, allora esso non ha nulla all'interno; è un guscio comunicante. Se c'è un interno esso coincide con i messaggi che si scambiano con l'ambiente. Immagine e forma sono più importanti di una sostanza che non c'è più, per un uomo totalmente eterodiretto; l'uomo si guida dal di fuori, dipende dall'esterno e dalla qualità dei suoi scambi, in virtù (esclusiva) della sua capacità di trattare ed elaborare l'informazione che gli è necessaria per mantenersi vivo.

È bene precisare che questa "rinuncia all'interno" non corrisponde affatto per Wiener a una rinuncia al pensiero. I processi "mentali" sono tutto ciò che distinguono uomini e computer da altri esseri viventi più istintuali. Wiener, $\mathrm{i}$ cibernetici e i primi scienziati a occuparsi di computer in senso moderno come Turing e Von Neumann, parlando di pensiero si riferiscono al ragionamento, e al ragionamento come calcolo. In quanto tale, il pensiero è qualcosa di cui si possono esprimere le leggi, valide in generale; e in quanto essere privo di interiorità, il pensiero non appartiene all'uomo, ma consiste nel flusso di informazioni che costituisce il mondo. Ecco allora emergere il mito della macchina, la quale può garantire, a differenza degli umani, un numero esiguo di errori nella trasmissione di "pensiero"; ecco la volontà di fare delle macchine i nuovi "decisori” della società:

Le macchine per la comunicazione, in quest'ottica, non servono soltanto a trattare e a scambiare individualmente informazione. Esse partecipano alla corrente globale di lotta contro l'entropia, mantenendo aperti i canali di comunicazione. Sono dunque comunicazione in atto - poiché permettono d'intervenire sul mondo e hanno quindi una funzione politica e sociale - e al tempo stesso la concretizzazione del sapere relativo alla comunicazione. (Breton 1992: 32 tr. it.)

Le diverse parti della macchina devono parlare l'una all'altra con un appropriato linguaggio, senza rivolgersi o ascoltare persona alcuna, salvo che nella fase iniziale e in quella finale. Possiamo dire questo è il nuovo contributo per rendere generalmente accettata l'estensione alla macchina del concetto di comunicazione. (Wiener 1950: 191192 trad.it)

Gli uomini sono al servizio della macchina affinché essa possa essere al servizio degli uomini. Gli sforzi degli "uomini di buona volontà" devono tendere dunque a fare delle macchine "esseri" completamente capaci di prendere decisioni; poi esse renderanno il mondo più ordinato, quindi migliore: si tratta di dispositivi, come gli organismi viventi ma più efficienti, in grado di offrire una strenua resistenza sia pur locale e temporanea alla tendenza al disordine, producendo isole di organizzazione fatte di "buona informazione".

Naturalmente, la funzione di autoregolazione che Wiener si attende dalla diffusione e amplificazione delle macchine e dei canali comunicativi ha un diretto impatto sulle future forme politiche che la società dovrà darsi. L'ideale di Wiener è anarchico, ma fondato sulla ragione e sul calcolo; se pure vagheg- 
Eduardo Grillo | L'utopia della comunicazione

gia uno Stato mondiale, non c'è niente nella sua "profezia utopica" che possa essere ricondotto a un forma di governo. Il compito degli uomini sarà quello di "liberare" l'informazione costruendo reti comunicative estese ed efficienti; in tal modo la società interconnessa si autoregolerà, in virtù delle decisioni ordinate delle macchine.

Anarchismo, trasparenza, affermazione dell'uomo in se stesso: lo abbiamo visto; si tratta di ideali di "liberazione". Ma non sfugge il prezzo da pagare, i mezzi attraverso i quali l'utopia potrà rendersi concreta. L'appello continuo alla natura formale dell'uomo, alla lotta al disordine, alla razionalizzazione dei mezzi e dei fini doveva suonare alquanto costringente a un'intera generazione di artisti. Eppure, lo vedremo, informale non è informe; l'arte non si limitò a sposare la causa o a reagire alla proposta di forme ordinate; piuttosto, si trattò di un'ampia elaborazione, che fece leva anche su esperienze scientifiche diverse.

\section{La mossa dell'Informale}

Negli stessi anni, il mondo dell'arte viene segnato dal fenomeno dell'Informale. Se l'etichetta appare ambigua, l'uso che ne è stato fatto lo è ancor di più; pertanto è necessario chiarirne il senso, e soprattutto l'uso che se ne vuol fare. Un modo per rispettare le diversità individuali e contemporaneamente salvaguardare l'unità degli intenti e degli esiti è considerare l'Informale non come una categoria critica ben definita, ma come tendenza espressiva generale in un dato periodo.

Nell'assumere questa prospettiva, Eco faceva rientrare l'arte informale all'interno della poetica dell'opera aperta (Eco 1976: 153). Come è noto, tutte le opere sono in qualche modo "aperte" all'interpretazione del fruitore; con questa espressione, Eco voleva invece indicare un accentuarsi del carattere ambiguo del "messaggio" artistico tipico delle poetiche contemporanee. Si tratta di una "proposta di un "campo" di possibilità interpretative, come configurazione di stimoli dotati di una sostanziale indeterminatezza, così che il fruitore sia indotto a una serie di "letture" sempre variabili; struttura, infine, come "costellazione" di elementi che si prestano a diverse relazioni reciproche» (Eco 1976: 154). È questa la prospettiva principale che si seguirà qui.

Il termine, com'è noto, fu trovato da Michel Tapié, nel contesto di un discorso globale sulla radicale diversità delle nuove forme espressive; un'arte autre, che tagliava i ponti con la storia:

[...] i pittori, con l'apparente libertà di una tecnica moltiplicabile all'infinito in nuove ricerche, agiscono deliberatamente senza di essa in un informel che si comporta nei confronti dell'abituale imperativo formale con la più indifferente disinvoltura e la più feconda anarchia. L'occidente scopre finalmente il Segno, e esplode nella veemenza di una calligrafia trascendentale, d'una iper-significanza ebbra della crudele vertigine di un divenire allo stato puro. (Tapié 1952: 5)

Il passo ruota intorno alle seguenti parole-chiave, forse riconducibili a coppie: disinvoltura - anarchia, segno - divenire. Disinvoltura nei confronti del canone: gli artisti non si limitano a mettere in discussione i movimenti che 
Eduardo Grillo | L'utopia della comunicazione

avevano dominato il Novecento fino a quel momento, ossia le Avanguardie Storiche, ma "saltano" a piè pari (o almeno questa era l'intenzione) il momento del dialogo. L'eredità del passato è guardata con insofferenza; pertanto l'Informale si caratterizza come

un complesso di ricerche e di fermenti che hanno quale comune denominatore l'impegno [...] di superare le vecchie concezioni idealistiche, spiritualistiche e razionaleggianti della Forma, e tanto l'immagine astratta come l'entità eidetica e trascendente del fenomeno, quanto l'immagine naturalistica come effigie del mondo, riferibile ai fenomeni ma distinta da essi, per vagliare le possibilità ulteriori, altre, di una forma che si proponga essa stessa come fenomeno. (Calvesi 1966: 99)

Si tratta della volontà di proporre un modo di fare arte come forma di vita, tentando di spezzare il legame rappresentativo, che si poneva come riflessione sulla vita, restando distinta da essa. Ciò che emerge dalle opere è dunque la situazione esistenziale, soggettiva, non tanto un punto di vista quanto l'espressione di una reazione personale alle circostanze, tragiche in quegli anni, in cui toccava in sorte di vivere (cfr. Crispolti 2005).

Così, dal 1945 gli artisti coinvolti nella "corrente" informale si situano in modo quasi automatico oltre le opposizioni fondamentali (forma-contenuto, astratto-concerto) che avevano accompagnato e le realizzazioni artistiche e le riflessioni estetiche. Ad avvantaggiarsene è la materialità dell'opera, o la materia come soggetto assoluto; e con ciò arriviamo al segno. Segno inteso non tanto nel suo carattere di rimando, ma proprio come gesto, come traccia che il percorso della mano lascia sul supporto: «il punto di partenza è la superficie da animare - tela o foglio di carta - e la prima macchia di colore o d'inchiostro che vi si getta: l'effetto che si produce, l'avventura che ne risulta. È questa macchia, a mano a mano che la si arricchisce e la si orienta, che deve guidare il lavoro» (Dubuffet 1946)². Quindi: assenza di un piano predeterminato, di una forma-progetto; ma non radicale informità. L'informale non è senza forma, ma è mancanza di una forma precostituita, o imposta all'opera.

$\mathrm{Ma}$, se si tratta di opere tendenzialmente statiche nel loro supporto, piuttosto convenzionali nel loro proporsi all'apprensione dell'osservatore, quale movimento, quale indeterminazione vi si potrà rintracciare? Una più "matura e radicale",

perché qui veramente i segni si compongono come costellazioni in cui la relazione strutturale non è determinata, in partenza, in modo univoco, in cui l'ambiguità del segno non è ricondotta (come per gli impressionisti) a una riconferma finale della distinzione tra la forma e lo sfondo, ma il fondo stesso diventa il soggetto del quadro (il soggetto del quadro diventa lo sfondo come possibilità di metamorfosi continua). (Eco 1976: 157-158)

Questa interpretazione "gestaltica" è fondamentale; soggetto e sfondo si confondono nell'Informale, si sovrappongono, uno invade lo spazio dell'altro.

2 La traduzione italiana del passo è tratta da (Vergine 1999: 15). La traduzione integrale dell'articolo è disponibile nella raccolta curata da Renato Barilli e pubblicata nel 1971. 
Eduardo Grillo | L'utopia della comunicazione

Più precisamente, lo sfondo sembra generare il proprio processo di trasformazione, come una riserva di possibilità da cui emergono le sue linee di sviluppo, come la chora, il territorio, il terreno inclusivo su cui si esercita la vita:

Senza essere ancora una posizione che rappresenta qualcosa per qualcuno, vale a dire senza essere un segno, la chora è nondimeno una posizione che rappresenta qualcuno per un'altra posizione, vale a dire che non è ancora un significante, ma si genera in vista di una tale posizione significante. Né modello né copia, è anteriore e soggiacente alla figurazione e dunque alla speculazione e non tollera analogie che non il ritmo vocale o cinesico. [...] Siamo qui in una modalità della significanza dove il segno linguistico non è ancora articolato come assenza d'oggetto e come distinzione tra reale e simbolico. (Kristeva 1974: 22 tr. it.) ${ }^{3}$

Tuttavia, non si deve concludere che l'informale rinneghi la dimensione formale; essa non viene ignorata, ma lavorata in vista di un'apertura. Si tratta di un indugiare sulla soglia stessa della possibilità:

In questo senso quindi Informale vuol dire negazione delle forme classiche a direzione univoca, non abbandono della forma come condizione base della comunicazione. L'esempio dell'Informale, come di ogni opera aperta, ci condurrà dunque non a decretare la morte della forma, ma una più articolata nozione del concetto di forma, la forma come campo di possibilità. (Eco 1976: 181-182)

Vediamo qui profilarsi una convergenza e insieme una presa di distanza dalla diffusione della nozione di comunicazione della cui origine ci ha parlato Breton. Anche qui infatti abbiamo un'intenzione comunicativa; non solo, abbiamo la volontà di tenere aperta la comunicazione, sebbene spingendo al limite le sue possibilità. Mentre Wiener e i primi teorici della comunicazione insistevano sulla necessità di ridurre il "rumore" sovrapponendo un "sistema di probabilità" a una situazione di equiprobabilità, l'intento dell'informale è quello di assestarsi sulla soglia della condizione di indecidibilità, al di qua della determinazione. Gli artisti informali tentano di portare il dialogo su un livello in cui ciò che conta è la vitalità che orienta il gesto, esplorando le vie offerte dalla materia in ogni direzione. Il fruitore ideale avrà il compito di seguirne il plurimo svolgimento.

Ora, a ben guardare la preoccupazione degli Informali è la stessa di Wiener; egli partiva da un'esigenza di apertura dei canali ove potesse "scorrere" l'instancabile flusso delle informazioni. Diverso è l'atteggiamento nei confronti della realizzazione di questo "ideale"; dove Wiener, impressionato dagli orrori dei primi anni Quaranta, si appellava alla razionalizzazione dei processi decisionali e comunicativi, gli artisti informali si astengono dall'imporre una razionalità, lasciando il campo delle scelte aperto e indefinito.

Non sfugge infatti l'impatto degli eventi tragici della seconda guerra mondiale sulla poetica informale. La temperie artistica del secondo dopoguerra è segnata, a volte in modo esplicito, dal disorientamento soggettivo dovuto

3 Su questi temi, e non solo, rimando al preziosissimo Milani (2015). 
Eduardo Grillo | L'utopia della comunicazione

all'impotenza della ragione sulla comprensione della realtà, nonché sulla incertezza della condotta da adottare. Per l'Informale sarebbe fuorviante limitarsi a parlare di una liberazione delle tecniche espressive dal peso della tradizione; si trattò piuttosto di

un gesto necessario per radicalizzare il "grado zero" a cui era giunto il linguaggio a causa della violenza della storia. Infatti la crisi post-apocalittica del secondo dopoguerra sembrava aver fatto "tabula rasa" di ogni possibilità espressiva, dissolvendo i legami tra uomo e mondo in un caotico magma di esperienze prive di senso (Milani 2005: 204).

L’incapacità di rendere conto della barbarie che si era appena consumata rendeva cioè indispensabile lavorare alla rifondazione del linguaggio, ormai sospinto nel regno dell'ineffettualità. L'intento era di reagire alla condizione di perdita, senza rifugiarsi nel recupero di schemi che di fatto ormai risultavano inservibili. Si trattava piuttosto di proiettarsi verso il confronto con le inquietudini del tempo:

[...] è facile dimostrare come siffatta situazione dell'arte sia parallela all'instabilità morale e sociale, all'irrequietezza delle strutture, al pensiero relativo di questi anni, come un unico impulso muova tutte le forme...un segno grafico come quello di Wols descrive minutamente la storia e la condizione dell'uomo "effimero" con un'intensa, sconvolgente immediatezza [...] in ogni caso, l'inquietudine del nuovo, le diverse ipotesi tecniche, l'aprirsi sempre a infinite scoperte del linguaggio segnano, nel loro variare, il tempo con una energia che sgomenta. (Anceschi 1988: 40)

Nel suo costituire un'alternativa, la proposta informale sviluppa a modo suo alcune conseguenze delle recenti scoperte scientifiche. In questo senso, come tutte le opere aperte anche l'Informale si costituisce come metafora epistemologica; pur accettando le condizioni di partenza del mito di Wiener, sposta l'accento dalla forma formante, ordinata e vincolante, a quella indeterminata. Ma è opportuno chiarire cosa Eco intenda come metafora epistemologica, e poi valutare i punti di riferimento scelti dagli artisti del periodo in questione. Secondo Eco, tutte le opere aperte consistono in

risoluzioni strutturali di una diffusa coscienza teoretica (non di una teoria determinata, ma di una persuasione culturale assimilata): rappresentano la ripercussione, nell'attività formativa, di determinate acquisizioni delle metodologie scientifiche contemporanee. (Eco 1976: 159). ${ }^{4}$

Si tratta cioè della trasposizione, o della libera elaborazione, delle assunzioni concettuali che la scienza del Novecento ha proposto al mondo della cultura. Come ci ricorda Eco, se è vero che gli innesti interdisciplinari vanno

4 Qui Eco utilizza l'espressione attività formativa in un precisa accezione, desunta da Pareyson: una sorta di immissione dell'attività spirituale e vitale nella figurazione, sotto la specie della costruzione organica, valida in generale per tutte le attività umane (che consisterebbero nella produzione di forme) e in particolare per il fare artistico. Per un supplemento d'inchiesta, rimando a (Pareyson 1988). 
Eduardo Grillo | L'utopia della comunicazione

sorvegliati e avanzati con cautela, è inutile rimproverare un artista o un'intera cultura di appropriarsene in modo indiscriminato o azzardato. Si tratta di un processo di appropriazione a scopi immaginativi dei portati dell'epoca. Così, Mathieu (1958) scrive un breve ma denso saggio in cui stabilisce un parallelo tra il crollo dei valori classici nell'arte e l'emersione dei concetti di indeterminazione e di accettabilità della contraddizione, la nuova attualità del concetto di entropia, il fallimento almeno parziale delle nozioni collegate allo spazio, al tempo e alla gravitazione provenienti dalla fisica del tempo. L'intento dell'artista è di disegnare una sorta di sviluppo delle società occidentali che segue lo slittamento dall'ideale al possibile, passando per il reale e l'astratto. Si noterà la sensibilità di Mathieu nel fare appello alle acquisizioni scientifiche più recenti, che fanno diretto riferimento anche alla temperie cibernetica. Soltanto che laddove Wiener vedeva l'entropia come un nemico da contrastare con ogni mezzo, Mathieu intravvede la possibilità di abbracciare il misticismo e di tendere verso nuove forme trascendentali di espressione.

Tra la tensione verso la trascendenza di Mathieu e l'appello alla disciplina di Wiener, la silloge di Eco è preziosa, sia per chiarire il senso della metaforica epistemologica, sia per l'accurata descrizione dell'arte degli anni '50:

Di qui la funzione di un'arte aperta quale metafora epistemologica: in un mondo in cui la discontinuità dei fenomeni ha messo in crisi la possibilità di una immagine unitaria e definitiva, essa suggerisce un modo di vedere ciò che si vive, e vedendolo accettato, integrarlo nella propria sensibilità. Un'opera aperta affronta appieno il compito di darci un' immagine della discontinuità: non la racconta, la è. Mediando l'astratta categoria della metodologia scientifica e la viva materia della nostra sensibilità, essa appare quasi una sorta di schema trascendentale che ci permette di capire nuovi aspetti del mondo. (Eco 1976: 159)

Tutti gli artisti coinvolti nella corrente informale erano insomma fortemente colpiti dalla barbarie, sollecitati dalle nuove direzioni della scienza, e vagheggiavano una nuova società in grado di segnare una decisa discontinuità con il passato recente, in nome dei valori di libertà e di condivisione; esattamente come i cibernetici. La differenza è questa: l'immaginazione wieneriana insisteva sul controllo, sui processi decisionali, sulla "lubrificazione" dei canali che lasciati liberi di connettersi avrebbero assicurato un futuro regolato e organizzato; l'utopia dell'informale è di segno inverso. Forme non strutturate, o poco strutturate, che puntano tutto sulla disponibilità non strettamente limitata dell'informazione, che rifiutano di imporre un sistema di probabilità riduca le possibilità. Quello che sembra aver impressionato gli artisti è soprattutto la ferocia organizzativa del male durante la guerra; di conseguenza, non un nuova organizzazione, più razionale, è per loro la chiave, ma l'indeterminatezza, che lascia aperte le vie della comprensione empatica. Un modello di comunicazione affatto diverso, non riducibile in bit e pacchetti digitali di informazione, ma fondata sul comune sentimento di compassione.

Si potrebbe forse parlare di un differente modello di interazione propugnato dagli informali; ossia, in luogo di una logica comunicativa in senso forte, 
Eduardo Grillo | L'utopia della comunicazione

essi sembrano avanzare un modello conversazionale. ${ }^{5}$ Comunicare voleva dire in principio "mettere in comune" - non importa cosa; o anche trasmettere una notizia, o ragguagliare, ossia fornire i mezzi per un orientamento. Vi insistono due prospettive: una paritaria - la condivisione; l'altra asimmetrica: sono io (o sei tu) che ti do (mi dai) le notizie di cui si abbisogna per regolarsi. Questo secondo aspetto è magnificato dalla prospettiva di Wiener, o meglio ne rappresenta l'origine; da quel primo momento, nella dinamica iterativa degli scambi ognuno contribuisce a orientare gli altri, e ne è a sua volta orientato, fino a conseguire l'autoregolazione su base sociale.

Conversazione, invece, ha originariamente il significato di volgersi all'altro e trattenere; nella conversazione ci si in-trattiene, si stabilisce un legame autentico, e lo si coltiva. Partendo da una comune fonte - l'informazione, da in-formare, dare una forma - i cibernetici elaborano l'idea di una griglia, una struttura; gli informali verso un comune spazio di intendimento, un processo. In questo, gli artisti dell'Informale non abbandonano la forma; per questo, non la costringono e lasciano che sia la dinamica opera (aperta) - fruizione a svilupparla.

Per riassumere con un'immagine, potremmo dire che ci sono due soli che attraversano lo stesso cielo nell'immediato dopoguerra: il sole della forma formante, dove la riduzione dell'entropia è il primo e più importante obiettivo; e il sole della forma indeterminata, intesa come movimento generatore, dove il disordine è una risorsa da non soffocare, ma da sfruttare a pieno regime. Naturalmente, sono due immagini a cui si collegano due modelli diversi di società; per Wiener si trattava di curarsi dei mezzi, dei canali, per garantire l'avvento di una società armonica e razionale; per gli artisti, si trattava di liberare i linguaggi e le forme, per curarsi della società, non così razionale forse, ma non irrazionale: solidale (non informe: informale, elastica, e adattabile).

Anche il riferimento alle nuove acquisizioni scientifiche operato dagli artisti più inclini alla speculazione non va in direzione di una negazione dell'ordine, ma dell'inclusione del disordine; Mathieu (1958) è esplicito in proposito. Per disinnescare l'orrore, il cedimento agli impulsi, non resta che inglobarli in una forma aperta, che garantisca lo spazio sufficiente a farli sfogare. E non vi è nessun rifiuto della comunicazione; ma l'appello a un intendimento costruito per somma di differenze, per composizione delle spinte contradditorie, al fine di generare una nuova cornice, inclusiva e articolata.

\section{La fine dell'utopia e la Pop Art}

Il clima culturale (ed emotivo) che abbiamo tentato di ricostruire è il frutto di un periodo incerto, e per questo ancora sognante, progettuale. Esso è destinato, man mano che le società occidentali si assestano e prendono la loro direzione, a esaurirsi lentamente. Durante gli anni Sessanta si assiste a due fenomeni collegati ma opposti. Da un lato, la nascente società della comunica-

5 Sull'opposizione di queste due forme di interazione, vedi (Ronchi 2003). In questo volume, Ronchi affronta il modello comunicativo imperante, nato proprio nel periodo che ci interessa, contrapponendogli il modello di "comunicazione vivente" rappresentato dalla situazione d conversazione. 
Eduardo Grillo | L'utopia della comunicazione

zione si converte decisamente in "società dei consumi"; il boom economico si diffonde a macchia d'olio in tutta Europa, mentre si accentua il carattere ideologico e mercantile dei flussi comunicativi. Dall'altro lato, questo fenomeno pervasivo produce contemporaneamente i suoi "anticorpi", e nel volgere del decennio emerge lo spirito di contestazione che tutti conosciamo.

Mentre questo secondo aspetto viene soltanto accennato da Breton, egli insiste sullo stretto legame tra la trasformazione dell'utopia della comunicazione e l'affermazione del liberalismo come ideologia dominante, sebbene nascosta (nascosta è la sua natura di ideologia). In realtà, società liberale e società della comunicazione condividono soltanto alcuni fini, mentre altri aspetti sono in decisa opposizione, come l'individualismo esasperato della seconda e la tendenza al collettivo della prima. L'evoluzione di alcuni protagonisti tecnico-economici sono emblematici delle contraddizioni che segnano il percorso delle società della comunicazione; ad esempio,

i media, portatori di un'utopia della trasparenza, ma allo stesso tempo soggetti alla dialettica degli interessi politici ed economici, e le reti informatiche, in cui l'ideale utopico di un'informazione razionale liberamente circolante urta quotidianamente contro gli imperativi della proprietà privata e della separazione e distinzione fra i diversi gruppi sociali. Wiener che - ricordiamolo - non era un liberale, aveva previsto che se l'informazione fosse divenuta una merce, l'entropia contro la quale si pensava lottasse si sarebbe sviluppata in modo ancor più devastante. (Breton 1992: 115 trad.it)

Nonostante i timori di Wiener, è invece ciò che è successo. Gli anni '6o hanno messo al centro i consumi, esaltando il carattere di scambio del valore della comunicazione.

La società si mantiene, come avevano visto i teorici della comunicazione, attraverso lo scambio di simulacri degli oggetti, come sarà sempre più chiaro negli anni a venire: «Viviamo così al riparo dei segni e nella negazione del reale. [...] si può affermare che la dimensione del consumo, così come l'abbiamo qui definita, non è quella della conoscenza del mondo, ma neppure più quella dell'ignoranza totale: è quella del disconoscimento» (Baudrillard 1970: 213 tr.it).

Disconoscimento dovuto al passaggio dai bisogni e dalla loro soddisfazione, centrata sugli oggetti, all'istigazione dei bisogni, centrata sui simulacri. I media di massa divengono in quegli anni il punto di riferimento della vita culturale, la produzione di massa rende possibile il consumo di oggetti nuovi $\mathrm{o}$ in passato troppo costosi. L'effetto di questa sovrapposizione è la riduzione a intrattenimento di qualunque contenuto culturale, reso quasi necessario anche a causa del nuovo oggetto di interesse da parte delle classi dirigenti: il tempo libero. I media di massa si fanno sempre più carico di una rielaborazione e semplificazione della cosiddetta "cultura" alta offrendola in "formato famiglia". Anche quando l'operazione non ha intenti di livellamento, la messe di informazioni lanciata nello spazio pubblico è tale da non consentire un accurato discernimento; un prodotto equivale all'altro, considerato nella sua fungibilità nell'occupare lo stessa durata di una serata di riposo.

Si tratta della fine dell'utopia di Wiener. La liberazione dell'informazione, la moltiplicazione dell'universo comunicativo ha generato un incremento di 
Eduardo Grillo | L'utopia della comunicazione

entropia; l'ordine e la discriminazione si sono resi difficili e più faticosi, come pure lo stesso scienziato aveva previsto, paventando proprio uno sfruttamento mercantile. E gli oggetti da possedere non sono soltanto nuove comodità, ma cominciano ad essere investiti di valori identitari, una tendenza che andrà sempre più accentuandosi: un oggetto risponde certo a un bisogno, ma viene scelto per la sua capacità di "rappresentare" meglio il consumatore. Il che è evidentemente legato alla nuova forma che assume il fenomeno della comunicazione su scala sociale; non si veicolano più mere informazioni, ma immagini in cui specchiarsi.

In questo contesto si inserisce la Pop Art. Per delineare i contorni ideologici dell'arte pop, è opportuno mettere in evidenza il perdurare negli anni Sessanta della sensazione di smarrimento dell'uomo; solo che adesso non ci si trova più nel deserto post-bellico, ma in una giungla popolata da oggetti. Lo smarrimento cambia scenario; dall'indigenza e dall'ottundimento post-traumatico, all'abbondanza, al bombardamento delle immagini e delle informazioni. La Pop Art tematizza, più che elaborare, i nuovi rapporti tra soggetto, oggetto e regno semiotico, ossia la natura di segni delle forme artistiche e produttive (Cfr. Carriero 2003). La sovrapposizione tra società (post-)industriale e logica comunicativa ha l'effetto di disincarnare gli oggetti, seguendo un continuo slittamento tra valore d'uso, valore di scambio e valore segnico, ossia rappresentativo e posizionale; «La quotidianità pop è invece l'apparente differenziazione nella ripetizione: l'hamburger o la lattina di Coca Cola, isolati sulla tela, sono infatti l'estrema significazione del processo di svuotamento della quotidianità, esibita come pubblicizzata» (Carriero 2003: 28).

L'oggetto viene semplicemente esposto e contemporaneamente offerto, replicando la postura consumistica che gli era connaturata. Ma la rappresentazione sulla tela ha l'effetto di accentuare il processo di de-funzionalizzazione; in questo senso, Carriero ha indubbiamente ragione nel sottolineare l'esito di celebrazione dell'oggetto di consumo da parte della Pop Art, che da semplice correlato concreto di un bisogno diventa feticcio ${ }^{6}$.

L'utopia della comunicazione ha condotto quindi a un totale distacco dal mondo, invece di consentirne un più adeguato governo. $\mathrm{E}$ il versante della produzione artistica completa il processo di derealizzazione, portando alle estreme conseguenze la progressiva reclamizzazione della comunicazione, dando vita a una immaginifica feticizzazione.

All'ideale della riduzione dell'entropia si sostituisce l'ideale della globalizzazione del lavoro e dei consumi; in tal modo, la totale fungibilità degli esseri umani e il loro assorbimento nella catena sociale cambia di segno. Infatti, per Wiener si trattava di rendere gli uomini trasparenti e considerarli sostituibili per garantire flussi di comunicazione puliti, efficaci e razionali. Per la nuova "utopia" consumistica, l'obiettivo della fungibilità è soltanto effettuale, essen-

6 Questo rilievo ha il merito di mettere nella giusta evidenza lo strappo, già cominciato dagli artisti informali e ancor prima dalle avanguardie storiche, nei confronti del linguaggio dell'arte tradizionale, con la sua insistenza sui valori dell'irripetibilità e della modalità rappresentativa: «Il barattolo pop [...] non conosce alcun tempo trascorso o vissuto, non possiede un carattere individuale, ovvero non è personalizzato né appartiene a una dimensione artistica distinta da quella reale» (Carriero 2003: 36). 
Eduardo Grillo | L'utopia della comunicazione

do il nuovo modello di uomo teso certo verso l'efficienza, ma fondato sulla distinzione dei gusti: siate voi stessi, consumando come tutti gli altri. La sensibilità di Warhol e compagni di avventura ha consentito alla Pop Art di riassumere efficacemente il meccanismo di individualizzazione/massificazione del nuovo universo della comunicazione, mediato dalla funzione paradigmatica dello star system: «Il presidente beve Coca Cola [...] Liz Taylor beve Coca Cola e [...] anche tu puoi berla» (Wahrol 1975: 87 tr. it.).

\section{Conclusioni}

Poche parole per riassumere il percorso seguito fin qui. La tesi di Breton sulla nascita della società della comunicazione ci è sembrata un buon viatico sia per contestualizzare alcuni aspetti dell'impresa cibernetica, sia per comprendere la vocazione comunicativa del fenomeno dell'Informale.

Se è accettabile l'ipotesi di Breton, che colloca la nascita dell'utopia della comunicazione negli anni Quaranta e lo qualifica come valore post-traumatico emerso in seguito agli orrori della seconda guerra mondiale, allora le vie seguite dagli artisti informali si distaccano non tanto per i temi quanto per gli strumenti e i fini dalla nuova utopia. Il cenacolo cibernetico, al centro della ricostruzione di Breton, insisteva infatti sulla proposta degli sviluppi della comunicazione in vista di una prospettiva d'ordine e controllo di spinte irrazionali e processi sociali, sulla base di un'idea di uomo trasparente e senza interiorità.

Il fascino della macchina si spiega facilmente in un contesto di sconcerto per la tragica irrazionalità organizzata degli uomini, che si sono serviti della tecnica e della scienza per dare vita ai più terribili crimini contro l'umanità. La macchina non ha passioni, non è in grado di autoconvincersi della logica della propria follia. Ecco il bisogno di regolazione, di controllo, di ordine; ma ecco anche la voglia di liberazione, di lasciarsi alle spalle le asfissianti pratiche totalitarie, di un nuovo ordine elastico e soprattutto autenticamente umano.

L'arte informale risponde in fondo a queste esigenze, tuttavia proponendo un modello conversazionale. Ciò che caratterizza una conversazione informale è proprio l'adozione di uno stile che non sia costretto dall'etichetta, ma che lasci la possibilità di spaziare liberamente da un tema all'altro, mentre ci si in-trattiene. Le forme indefinite, che si stemperano, che rifuggono dalle forme chiuse e rigide sono in effetti l'appello al dialogo, alla disponibilità di adattarsi alle nuove condizioni di volta in volta, di dedicarsi alla conversazione senza schemi prefissati.

L’informale si eclissa proprio quando, con gli anni Sessanta, ma sempre di più in seguito, l'utopia informazionale e comunicativa cambia di segno. La tensione verso un mondo nuovo, migliore, assume i connotati del benessere, del possesso, del perfezionamento dei cicli produttivi e della disponibilità al consumo.

La Pop Art è il linguaggio artistico che s'impone in quegli stessi anni. Essa ha la sensibilità necessaria per cogliere la nuova direzione di un mondo che si affranca sempre più dal ricordo degli orrori passati. Ripetendo nelle sue opere un meccanismo produttivo che diviene sempre più capace di portare con sé 
Eduardo Grillo | L'utopia della comunicazione

nuove forme di vita, la Pop Art è l'interprete naturale della nuova utopia del benessere. La forma comunicativa dominante diventa la réclame, che lentamente soppianta le vecchie forme dell'Öffentlichkeit ${ }^{7}$; con essa, il conflitto tra il modello della comunicazione ordinata e quello conversazionale è ormai alle spalle, ma perché superato è il ritrarsi dall'orrore che ha caratterizzato i decenni precedenti.

La Pop Art contribuisce a sviluppare, mentre le sfrutta, le capacità di induzione identitaria del capitalismo consumista; $\mathrm{o}$, più prudentemente, le incarna perfettamente. Per questo si candida a rappresentare un mutamento che ha caratteri epocali: la comunicazione, mentre moltiplica sorgenti e canali, ripete il modello centralistico della produzione capitalistica, che scherma i bisogni con i desideri; laddove pochi anni prima, sia per i cibernetici sia per gli informali, erano ancora vive le speranze di una società libera e trasparente, in grado di fare i conti una volta per tutte con i suoi fantasmi.

\section{Bibliografia}

Anceschi L. 1988 [1958], «intervento», in Id. Interventi per «il verri» (1956-1987). Ravenna: Longo.

Arcangeli F. (1976), Dal Romanticismo all'Informale 1970-1971. Bologna : Alfa.

Bateson G. (1979) Mind and Nature. A Necessary Unit, E.P. Dutton, New York-Wildwood House, London-Bookwise, Sidney-Clarke, Irwin \& Co., Toronto-Vancouver (tr. it. Mente e Natura, Adelphi, Milano, 1984).

Baudrillard J. (1970), La société de consommation, Paris: Gallimard (tr. it. La società dei consumi, Bologna: il Mulino, [1976] 2010).

Breton P. (1992), L’utopie de la communication, Paris: La Découverte [II ed. 1995]. (tr. it. L'utopia della comunicazione, prefazione di Paolo Fabbri, Torino: Utet, 1995).

Calvesi M. (1966) [1963], «L’informale in Italia fino al 1957», in Id. Le due avanguardie. Milano: Lerici.

Carriero C. (2003), Il consumo della Pop Art: esibizione dell'oggetto e crisi dell'oggettivazione. Milano: Jaca Book.

Crispolti E. (2005), «Sulla radicalità esistenziale dell'Informale», in Informale. Jean Dubuffet e l'arte europea 1945-1970. Catalogo della mostra a cura di L. M. Barbero, Modena 18 dicembre 2005 - 9 aprile 2006, New York.

Dubuffet J. (1946) [1945], «Note pour les fins-lettré», in Id. Prospectus. Paris: Gallimard (tr. it. I valori selvaggi. Prospectus e altri scritti, a cura di Barilli R., Milano: Feltrinelli, 1971).

Eco U. (1972) (a cura di), Estetica e teoria dell'informazione. Milano: Bompiani.

Id. (1976) [1962] «L'opera aperta nelle arti visive», in Id. Opera Aperta, Milano: Bompiani, , 153-184.

Habermas J. (1962) Strukturwandel der Öffentlichkeit. Untersuchungen zu einer Kategorie der bürgerlichen Gesellschaft (Habil.), Neuwied (tr. it. Storia e critica dell'opinione pubblica, Laterza, Bari, 1971).

7 Habermas J. (1962). 
Eduardo Grillo | L'utopia della comunicazione

Heims S.J. (1977) «Gregory Bateson and the mathematicians: from interdisciplinary interaction to societal functions", Journal of the History of the Behavioral Sciences, 13(2), pp.141-59.

Id. (1991) The Cybernetic Group 1946-1953, MIT Press, Boston (MA) (tr. it. I cibernetici. Un gruppo e un’idea, Editori Riuniti, Milano, 1997).

Klein R.K. (2015) The Cybernetics Moment: Or Why We Call Our Age the Information Age, John Hopkins University Press, Baltimora (MD).

Kristeva J. (1974), La Revolution du langage poetique, Paris: Seuil (tr. it. La rivoluzione del linguaggio poetico, Venezia: Marsilio, 1979).

Mathieu G. (1958), «D'Aristotle à l'abstration lirique», L'œil: revue d'art mensuelle, $52,28-35$.

Milani F. (2015), «L'informale: una poetica disintegrata», L'Ulisse - Rivista di Poesia, Arti e Scritture. 18(2), pp. 202-214.

Pareyson L. (1988) [1954], Estetica. Teoria della formatività. Milano: Bompiani.

Ronchi R. (2003), Teoria critica della comunicazione. Milano: Bruno Mondadori.

Tapié M. (1952), Un art autre. Paris: Giraud.

Vergine L. (1999), L'arte in trincea. Lessico delle tendenze artistiche 1960-1990, Milano: Skira.

Warhol A. (1975), The philosophy of Andy Warhol. From A to B and Back Again. San Diego (CA): Harcourt Brace Jovanovich (tr. it. La filosofia di Andy Warhol. Milano: Bompiani, 2001).

Wiener N. (1948), Cybernetics or Control and Communication in the Animal and the Machine. Paris: Hermann \& Cies \& Cambridge (Mass): MIT Press (tr. it. La cibernetica. Controllo e comunicazione nell'animale e nella macchina. Milano: Il Saggiatore, 1968).

Id. (1950), The Human Use of Human Beings. Boston: Houghton Mifflin Company (tr. it. Introduzione alla cibernetica, Torino: Bollati Boringhieri, 1966).

Eduardo Grillo è dottore di ricerca in Semiotica e comunicazione simbolica. Ha insegnato filosofia del linguaggio ed è cultore della materia in Semiotica all'Università di Perugia. Si occupa di narratologia e semiotica dell'arte. È autore di Semiotica dell'investigazione (Carocci, 2014). 